Review Article

\title{
Genetic Algorithm and BP Neural Network for College Physical Education Teaching Evaluation
}

\author{
Shuxin Wang $\mathbb{D}$ \\ Sias University, Zhengzhou, Henan, China \\ Correspondence should be addressed to Shuxin Wang; 11138@sias.edu.cn
}

Received 19 March 2021; Accepted 25 May 2021; Published 31 May 2021

Academic Editor: Shah Nazir

Copyright (C) 2021 Shuxin Wang. This is an open access article distributed under the Creative Commons Attribution License, which permits unrestricted use, distribution, and reproduction in any medium, provided the original work is properly cited.

Physical education is considered as the essential part of health plan and also reflects the higher education system. A teacher with physical education is accountable to teach physical education and/or health education to the students. This study has proposed a new approach for teaching evaluation with college physical education in universities. By combining neural network with genetic algorithm (GA), the approach that we proposed can improve the general traditional BP network, such as global convergence and training time. The model uses MATLAB software for empirical research work. According to MATLAB simulation and experiments, it was revealed that the combining neural network with GA algorithm has effective application prospects for the teaching evaluation with college physical education in universities.

\section{Introduction}

At present, we always do the teaching evaluation through different methods and approaches, as the teaching quality has always been the focus of social attention. In China, we have a huge amount of higher education system in which a large number of students are studying. The evaluation of training quality of a university is an important issue in front of us which need proper evaluation mechanism. The quality evaluation system has been considered as an important part of education [1-5]. At present, many teaching quality evaluation systems are established according to the standards of ordinary colleges and universities level. In the current evaluation system, there are some problems for the evaluation of university education quality which includes the following. (a) The evaluation index system is not reasonable which cannot reflect the characteristics for university physical education. (b) The teaching evaluation index often contains many no quantitative factors, so in the evaluation, there are some complex nonlinear relationships between the input and output. So, it is hard to build precise mathematical models through a relationship. (c) The teaching quality evaluation methods, such as the absolute evaluation method, the relative evaluation method, the rating method, the comment method, the realistic method, and the comprehensive scoring method, are either too subjective, or simple mathematical operations (such as addition, subtraction, multiplication, and division) are used to evaluate the teaching effect, ignoring the nonlinear relationship between the evaluation indexes and the teaching effect, and the results are difficult to reflect the teaching quality; it is also difficult to be accepted by people [6-13]. (d) It is difficult to make an accurate evaluation by using traditional methods to evaluate the results of some indicators, and the calculation is complex, the solution is cumbersome, and these algorithms also lack of self-learning ability [14-16].

To overcome these problems, this study has proposed a novel approach for teaching evaluation with college physical education in universities. By combining neural network with genetic algorithm, the current approach can progress the general traditional BP network, such as global convergence and training time. The approach has used MATLAB software for experimental work. Based on the simulation and experiments, it was shown that combining the neural 
network with GA algorithm has operational applications for the teaching evaluation with college physical education in universities.

\section{BP Neural Network with Genetic Algorithm}

The following sections briefly show the details.

2.1. Genetic Algorithm. Genetic algorithm (GA) mainly includes selection, crossover, mutation, and other operations. By combining neural network with genetic algorithm, it not only makes use of the strong learning ability of neural network but also combines the global search characteristics of genetic algorithm.

In this paper, we introduce the GA into the BP neural network for the evaluation model, which is a further optimization application of BP neural network. Various applications of GA exist $[17,18]$.

\subsection{BP Neural Network System Model Based on Genetic} Algorithm. In BP neural network, we use the descent method as the learning rule. By adjusting the weight value of each layer, we try to minimize the square sum of network error. As shown in Figure 1, firstly, representative teaching quality evaluation indexes are selected as input information; then, we sent these evaluation indexes to the input layer, hidden layer, and output layer for training. We use the genetic algorithm to iteratively learn the network weights according to the training objective function to find the best weights. The network becomes a stable pattern evaluator, and then, the evaluation results can be output.

The number of input nodes of the model is $n$ (evaluation index of college physical education quality), the number of middle layer nodes is $n$, and the number of output layer nodes is 1 , which is the evaluation value of college physical education quality. The input layer node transmits the information directly to the middle layer node, so the output of the input layer node is equal to the input; the output information of the middle layer node is the input information of the output layer node, and the output layer has only one node, which receives the input of the middle layer node and outputs the evaluation results of the teaching quality:

(1) Input layer node $p_{i}, i=\{1,2, \ldots, n\}$, that is, $n$ represents teaching quality assessment

(2) Middle layer node

The input is

$$
I_{j}=\sum_{j=1}^{n} \omega_{i j} p_{i}
$$

The output is

$$
O_{j}=\frac{1}{\left\{1+\left[\left(\sum_{i=0}^{n} \omega_{i j} p_{i}\right)^{-1}-1\right]^{2}\right\}}=\frac{1}{\left[\left(I_{j}^{-1}-1\right)^{2}\right]}
$$

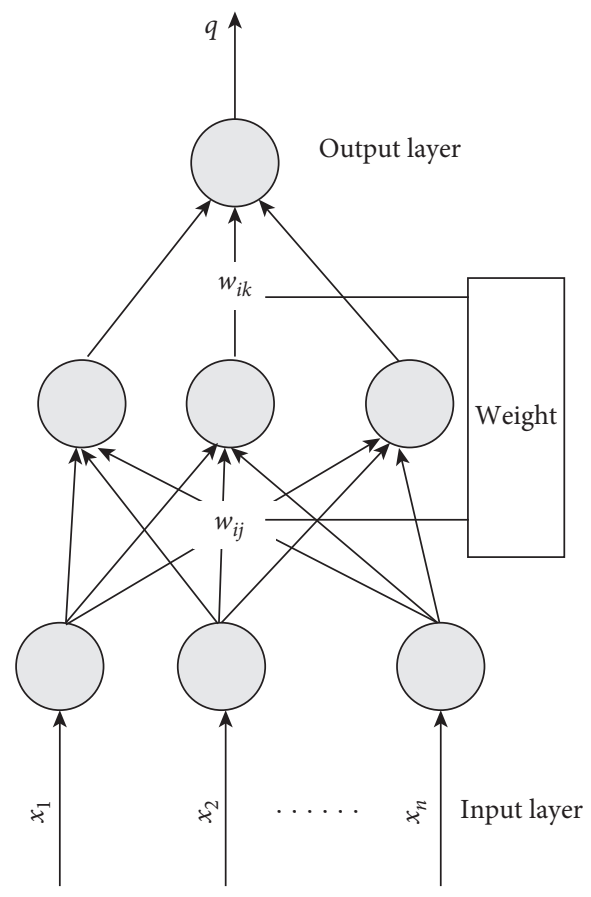

Figure 1: BP neural network training model with genetic algorithm.

where $\omega_{i}$ is the weight from the input layer node $i$ to the middle layer node $j$ and $p_{i}$ represents the $i$ th factor of the sample, i.e., the $i$ th teaching quality evaluation index.

(3) Output layer node: the output layer has only one node, and the input is the output of the middle layer node:

$$
q=\frac{1}{\left\{1+\left[\left(\sum_{i=0}^{n} \omega_{j} O_{j}\right)^{-1}-1\right]\right\}^{2}}
$$

where $q$ is the actual output value of the sample, that is, the calculated teaching quality evaluation value and $\omega_{j}$ is the connection weight from the middle layer node $i$ to the output layer node.

\subsection{Genetic Algorithm}

2.3.1. Coding Mode. The common genetic coding methods are real number encoding and binary encoding. Real number coding has high precision and is easy to search in a large space; binary coding is in line with the important biological characteristics of diploid chromosomes in higher organisms and has the function of long-term memory, which is convenient for various genetic operations. To facilitate genetic operation, binary coding is used for weight coefficient.

2.3.2. Training Objective Function. The training objective function is defined as the mean value of the squared sum of the error between the actual output and the evaluation value of $P$ samples: 


$$
E=\left(\frac{1}{p}\right) \sum_{p=1}^{p}[\bar{q}-q]^{2}=\left(\frac{1}{p}\right) \sum_{p=1}^{p} E_{j},
$$

where $P$ is the total logarithm of the training sample pattern, $q$ is the actual output value of the sample network, and $q$ is the expected value of the sample network. The actual output $Q$ of the identified object (the teaching quality evaluation model of Higher Vocational Colleges) is taken as the feedback signal, which is compared with the expected value of the neural network sample to make $E<e$. If $e$ does not meet the requirements due to the small number, the weight coefficient is adjusted continuously to meet the corresponding requirements.

\subsubsection{Weight Adjustment Model of BP Neural Network} Evaluation System. The purpose of network learning is to minimize $e$ by adjusting the connection weight in the network. Using the gradient descent method, the adjustment of connection weight is as follows:

$$
\left\{\begin{array}{l}
\omega_{i j}=-\eta\left(\frac{\partial E}{\partial v_{i j}}\right) \\
\omega_{j}=-\eta\left(\frac{\partial E}{\partial w_{i j}}\right) .
\end{array}\right.
$$

Among $\eta$, for the learning rate, $0<\eta<1$. Then, the connection weight adjustment amount between the input layer node and the middle layer node is

$$
\omega_{i j}=x_{i} w_{j} O_{j}^{2}\left[1-\sum_{i=1}^{n} \omega_{i j} x_{j}\right] \delta_{j} .
$$

The adjustment amount of connection weight is as follows:

$$
w_{j}=q^{2} O_{j}\left[1-\sum_{j=1}^{l} w_{j} O_{j}\right][\bar{q}-q]^{2} .
$$

By using the above model, the connection weight of the neural network can be determined according to the iterative algorithm of the common neural network, and the error between the actual output and the sample value can be reduced.

\subsubsection{Description of Connection Weight Optimization Problem Based on Optimal Neural Network.}

$$
\min (E)=f\left(w_{1}, \ldots, w_{n}\right),
$$

where $e$ is the total error of network training and $w_{1}, \ldots, w_{n}$ are the continuous weights after unified numbering which include the connection weights of input layer nodes and middle layer nodes and the connection weights of middle layer nodes and output layer nodes, $n$ is the total number of connection weights. The constraints are $a_{1} \leq w_{i} \leq b_{1}$. Among them, $b_{1}$ and $a_{1}$ are variables of $w_{i}$ of the upper and lower bounds of change.
2.3.5. Generation of Initial Population. As for the selection of initial population size, according to the research, the larger the nonlinearity of the problem, the larger the $P$. In this paper, $P=30$, and the initial population is composed of $P$ randomly generated strings.

2.3.6. Calculation of Fitness. The calculation of population fitness function is always a bottleneck problem in the application of genetic algorithms. Because the selection probability of individuals is directly proportional to fitness, the definition of fitness function has a great influence on the genetic algorithms. There is a close mapping relationship between the fitness function and objective function, so the following fitness calculation method is adopted:

$$
f_{i}= \begin{cases}C-E & E<C, \\ 0 & E \geq C,\end{cases}
$$

where $e$ is the training objective function and $C$ is the maximum of all $e$ in the current generation.

\subsubsection{Genetic Manipulation Produces New Populations.} Genetic manipulation mainly includes replication, crossover, and mutation. The purpose of copy operation is to improve the system global convergence and convergence efficiency. The main idea is that the replication probability $\mathrm{PR}$ of the string is proportional to its fitness, that is, the individuals with high fitness will be copied to the next generation with high probability. Cross operation can combine new individuals and search effectively in strand space to avoid effective gene deletion. Only in the case of effective gene deletion, the effect of mutation operation is more obvious. To take into account the efficiency of global convergence and avoid premature convergence caused by effective gene deletion, the following strategies are adopted in the selection of genetic operation parameters:

$$
P_{r}= \begin{cases}2\left(\frac{f_{1}}{C}\right)^{2}, & \frac{f_{1}}{C} \in[0,0.5], \\ {\left[1-2\left(1-\left(\frac{f_{1}}{C}\right)^{2}\right)\right],} & \frac{f_{1}}{C} \in[0.5,1],\end{cases}
$$

where $C$ is the parameter selected to define the fitness and $f_{1}$ is fitness. Equation (10) makes the replication probability change with the change of fitness, that is, the individuals with high fitness will be inherited to the next generation with a higher probability and the individuals with low fitness will be inherited to the next generation with a lower probability. The results show that the crossover probability PC is usually $[0.65,0.9]$, while the mutation probability $\mathrm{PM}$ is usually $[0.001,0.1]$.

\subsection{The Steps of Genetic Algorithm Training Are Neural Network Algorithm}

(1) The training sample data is selected, and the connection weights of input layer and middle layer and 
middle layer and output layer are generated randomly: $\omega_{i j}$ and $\omega_{j}$

(2) The actual output $Q$ of the sample is calculated according to equations (1)-(3)

(3) According to equation (4), calculate the value function $e$ of the square sum of the error between the actual output value and the expected value

(4) If $e \leq e$ meets the error requirements, the training is over, and go to step (11); otherwise, go to step (5)

(5) The weight adjustment values of the input layer and the middle layer and the middle layer and the output layer are calculated according to equations (6) and (7)

(6) Recalculate the new connection weight according to equations (9) and (10): $\omega_{i j}$ and $\omega_{j}$

(7) According to the new weights and sample data, the average error $e$ of the actual output $Q$ network of samples is recalculated according to equations (1) $-(4)$

(8) If $E \leq \delta$ and the error requirement is met, the training is over, and go to step (11); otherwise, go to step (9)

(9) Set the population size $n$, weight change range, crossover probability PC, and mutation probability $\mathrm{PM}$, and determine the replication probability PR according to equations (10) and (11)

(10) Copy crossover, mutation, and genetic algorithm calculation, according to the network weight generated by the genetic algorithm, as the initial weight value of the next training of the neural network model, and go to step (5)

(11) At the end of training, output the weight that meets the training accuracy: $\omega_{i j}$ and $\omega_{j}$

(12) According to the determined weight values $\omega_{i j}$ and $\omega_{j}$, put the experimental data to be evaluated and output the evaluation results

By fixing the weights of the neural network that has completed the training task, the network model constitutes a pattern evaluator.

\section{Evaluation of Physical Education Teaching Quality in Universities}

With the expansion of science and information technology, the applications of computer technology have been extensively adopted in all aspects of life. Class room teachings and various sports activities are enriched with the support of computer technology [19]. Various research studies have been conducted associated to physical education. A study has analyzed 35 physical education teachers' appreciation of a continuous professional development training embedded in self-determination theory, need-supportive teaching, and using quantitative and qualitative methods [20]. The application of virtual reality and web embedded system in the field of evaluation of physical education teaching is used
[21]. The study has evaluated the efficacy of need-supportive teaching in physical education on daily moderate-to-vigorous physical activity of girls through the mixed method evaluation [22].

\subsection{Establishing the Evaluation Index Teaching Quality.} In order to evaluate the teaching quality more scientifically and effectively, we must first determine a set of scientific evaluation index systems of teaching quality. On the basis of interviews with some teachers and students of Sias University, Zhengzhou University of Industrial Technology, Huanghuai University, and Huanghe Science and Technology University, combined with the summary of the theory and practice of the research on the evaluation of the quality of physical education teaching in universities, this paper establishes the evaluation index system of the quality of physical education teaching in universities, as shown in Table 1. From Table 1, we know the index system determines the network structure of the teaching-quality evaluation model.

3.2. Normalization of Input Index. Because the secondary index input is obtained by using the percentile system and scoring by students, the magnitude of each component value is very different. If the original data is applied directly without any transformation, the absolute value of the original data may be too large and fall outside the effective processing range of neurons, resulting in the so-called "saturation phenomenon." Even if the absolute value of the original data is not too large, it is possible that one component is too large, and the impact on the network is far greater than that of other components so that other components lose the ability to regulate the network. Therefore, it is necessary to normalize the input samples of the neural network. In this paper, the maximum and minimum method is used for normalization processing because this method is a linear transformation for data processing, which can better retain its original meaning and will not cause information loss. For the input data, the normalization formula used in this paper is as follows:

$$
X=\frac{I-I_{\min }}{I_{\max }-I_{\min }},
$$

where $x$ is the input value of the neural network after normalization, $I$ is the input value of the neural network without processing, $I_{\max }$ is the maximum input of neural network, and $I_{\min }$ is the minimum input of neural network.

3.3. Preparation of Sample Library. In the GABP neural network, based on the summary and analysis, according to the evaluation index of teaching quality, this paper formulates the teaching questionnaire of higher vocational colleges so that the students in our college can choose and score the indicators of teachers. The collected sample data were standardized according to formula (11). These data are transformed into data $[0,1]$. The processed sample data is shown in Table 2. 
TABLE 1: Evaluation index system of college physical education teaching quality.

\begin{tabular}{|c|c|c|c|c|c|}
\hline $\begin{array}{l}\text { First-level } \\
\text { indicators }\end{array}$ & Secondary indicators & Input & First-level indicators & Secondary indicators & Input \\
\hline Teaching attitude & $\begin{array}{l}\text { Rigorous lesson preparation } \\
\text { Operation wholesale }\end{array}$ & $\begin{array}{l}\mathrm{X} 1 \\
\mathrm{X} 2\end{array}$ & Content of courses & $\begin{array}{l}\text { Large proportion of practical training } \\
\text { Emphasis }\end{array}$ & $\begin{array}{l}\mathrm{X} 10 \\
\mathrm{X} 11\end{array}$ \\
\hline \multirow{7}{*}{ Lecture ability } & Content system & $\mathrm{X} 3$ & \multirow[t]{2}{*}{ Teaching method } & $\begin{array}{l}\text { Teach students in accordance with their } \\
\text { aptitude }\end{array}$ & $\mathrm{X} 12$ \\
\hline & Complex problems & $\mathrm{X} 4$ & & Focus on inspiration & $\mathrm{X} 13$ \\
\hline & $\begin{array}{l}\text { Integrating theory with } \\
\text { practice }\end{array}$ & $\mathrm{X} 5$ & \multirow{3}{*}{$\begin{array}{l}\text { Teaching and } \\
\text { educating }\end{array}$} & Interaction between learning and students & $\mathrm{X} 14$ \\
\hline & Heuristics & $\mathrm{X} 6$ & & Be a model for others & $\mathrm{X} 15$ \\
\hline & Key point and difficulty & $\mathrm{X} 7$ & & Strict requirements & $\mathrm{X} 16$ \\
\hline & Demonstration & $\mathrm{X} 8$ & \multirow{2}{*}{ Teaching effectiveness } & Students should master it well & $\mathrm{X} 17$ \\
\hline & Students' enthusiasm & X9 & & Students are good at doing things & $\mathrm{X} 18$ \\
\hline
\end{tabular}

TABLE 2: Training sample set after data preprocessing.

\begin{tabular}{lcccccccccccccc}
\hline No. & 1 & 2 & 3 & 4 & 5 & 6 & 7 & 8 & 9 & 10 & 11 & 12 & 13 \\
\hline X1 & 0.51 & 0.69 & 0.65 & 0.66 & 0.68 & 0.55 & 0.56 & 0.98 & 0.98 & 0.70 & 0.88 & 0.50 & 0.51 & 0.71 \\
X2 & 0.53 & 0.52 & 0.55 & 0.96 & 0.30 & 0.53 & 0.95 & 0.80 & 0.95 & 0.51 & 0.93 & 0.59 & 0.91 & 0.55 \\
X3 & 0.70 & 0.96 & 0.88 & 0.65 & 0.31 & 0.45 & 0.80 & 0.6 & 0.72 & 0.68 & 0.67 & 0.69 & 0.30 & 0.65 \\
X4 & 0.73 & 0.70 & 0.59 & 0.67 & 0.31 & 0.49 & 0.70 & 0.61 & 0.70 & 0.67 & 0.87 & 0.69 & 0.30 & 0.56 \\
X5 & 0.52 & 0.96 & 0.97 & 0.51 & 0.55 & 0.45 & 0.51 & 0.34 & 0.95 & 0.48 & 0.93 & 0.48 & 0.31 & 0.59 \\
X6 & 0.98 & 0.97 & 0.98 & 0.98 & 0.33 & 0.51 & 0.71 & 0.32 & 0.75 & 0.72 & 0.91 & 0.67 & 0.33 & 0.54 \\
X7 & 0.70 & 0.72 & 0.68 & 0.69 & 0.51 & 0.52 & 0.51 & 0.51 & 0.69 & 0.53 & 0.69 & 0.75 & 0.49 & 0.49 \\
X8 & 0.51 & 0.69 & 0.93 & 0.75 & 0.69 & 0.48 & 0.68 & 0.48 & 0.71 & 0.67 & 0.76 & 0.71 & 0.48 & 0.49 \\
X9 & 0.96 & 0.95 & 0.95 & 0.95 & 0.53 & 0.51 & 0.72 & 0.69 & 0.92 & 0.75 & 0.95 & 0.65 & 0.51 & 0.67 \\
X10 & 0.71 & 0.98 & 0.96 & 0.65 & 0.48 & 0.69 & 0.72 & 0.69 & 0.92 & 0.75 & 0.95 & 0.65 & 0.51 & 0.68 \\
X11 & 0.78 & 0.68 & 0.69 & 0.72 & 0.66 & 0.65 & 0.60 & 0.68 & 0.70 & 0.82 & 0.98 & 0.64 & 0.54 & 0.69 \\
X12 & 0.96 & 0.93 & 0.99 & 0.89 & 0.81 & 0.49 & 0.50 & 0.56 & 0.89 & 0.47 & 0.93 & 0.51 & 0.33 & 0.49 \\
X13 & 0.96 & 0.97 & 0.91 & 0.92 & 0.31 & 0.33 & 0.71 & 0.31 & 0.71 & 0.71 & 0.71 & 0.71 & 0.31 & 0.71 \\
X14 & 0.72 & 0.69 & 0.68 & 0.69 & 0.53 & 0.72 & 0.65 & 0.51 & 0.71 & 0.67 & 0.69 & 0.73 & 0.51 & 0.51 \\
X15 & 0.70 & 0.71 & 0.88 & 0.71 & 0.59 & 0.68 & 0.55 & 0.75 & 0.72 & 0.65 & 0.41 & 0.71 & 0.31 & 0.69 \\
X16 & 0.70 & 0.02 & 0.65 & 0.68 & 0.66 & 0.71 & 0.89 & 0.65 & 0.75 & 0.73 & 0.59 & 0.49 & 0.28 & 0.69 \\
X17 & 0.93 & 0.70 & 0.89 & 0.73 & 0.67 & 0.65 & 0.67 & .071 & 0.71 & 0.75 & 0.68 & 0.72 & 0.27 & 0.72 \\
X18 & 0.71 & 0.92 & 0.74 & 0.98 & 0.30 & 0.32 & 0.63 & 0.58 & 0.89 & 0.71 & 0.70 & 0.69 & 0.31 & 0.69 \\
\hline
\end{tabular}

3.4. Simulation Process and Results. The neural network toolbox in MATLAB contains the realization of various neural network algorithm programs. The realization of BP neural network by MATLAB generally has four steps: the first is the initialization of the network, the second is the creation of the network, the third is the simulation of the network, and the fourth is the training of the network. To test the performance of GA neural network training model, read the training data and teacher data (the first 10 groups of data in Table 2), generate the corresponding network model according to the neural network model structure proposed in Section 2.2, and set the error precision of network training as $e=0.001$. The operation parameters of the neural network are set as follows: learning efficiency 0.9 and momentum coefficient 0.9 , and the operation parameters of genetic algorithm are set as follows: weight variation range 0.5 , crossover probability 0.75 , and mutation probability 0.005 . The simulation process is as follows.

\subsubsection{Simulation Process}

Fid = fopen(train_data.txt);
$P=$ fscanf(fid, $\%$ g, $[10,18]) ;$

$\% P=$ mydata

$T=$ fscanf(fid,\%g, $[1,10]$ );

Net $==$ newff( $(0,1],[1,7],\{$ 'tansig','tansig' $\}$, traingdm');

Net.trainParam.show $=50$;

$\ldots$

net.trainParam.goal $=0.001$;

fidt = fopen('test_data.txt');

while(feof(fidt))

$Q=$ fscanf(fidt, $\% g,[4,18])$;

$B=\operatorname{sim}($ net, $\mathrm{Q})$;

End

Table 3 shows the results with BPGA, and the simulation evaluation results and expert evaluation results of the four test sets (the last four groups of data in Table 2 are shown in Table 4). From Tables 3 and 4, we know that it is not only the training samples which are very close to the expert evaluation results but also the simulation evaluation results of the four test sets are very close to the expert evaluation results. 
TABLE 3: Comparison of neural network training results based on genetic algorithm and actual evaluation results.

\begin{tabular}{lcccccccccc}
\hline Number & 1 & 2 & 3 & 4 & 5 & 6 & 7 & 8 & 9 \\
\hline Expert evaluation & 0.700 & 0.750 & 0.910 & 0.710 & 0.450 & 0.571 & 0.680 & 0.520 & 0.850 & 0.650 \\
Network test & 0.710 & 0.740 & 0.711 & 0.720 & 0.464 & 0.573 & 0.670 & 0.0490 & 0.860 & 0.651 \\
\hline
\end{tabular}

TABLE 4: Comparison between test results of the test set and actual evaluation results.

\begin{tabular}{lcccc}
\hline Number & 11 & 12 & 13 & 14 \\
\hline Expert evaluation & 0.825 & 0.630 & 0.511 & 0.630 \\
Network test & 0.810 & 0.651 & 0.535 & 0.610 \\
\hline
\end{tabular}

\section{Conclusions}

The contribution of the paper is to improve the traditional BP neural network using the GA. As the GA can iterative the weight factor to change the input of BP neural network. The basic idea is to use GA to optimize the input parameters of the neural network and speed the system convergence, finally, taking the optimization result as the initial value of BP algorithm, and then, train the network with BP algorithm. In this way, BP algorithm with GA is alternately run until the required accuracy of the problem is reached. In the experiment, we use many groups of sample data of our college to test and establish a BP neural network model based on genetic algorithm. Through the optimization of neural network connection weights, that is, the genetic evolution of the initial population generation by generation, the evaluation of college physical education teaching quality is realized. Results show the effectiveness of the study.

\section{Data Availability}

The data used to support the findings of this study are included within the article.

\section{Conflicts of Interest}

The author declares that there are no conflicts of interest regarding the publication of this paper.

\section{Acknowledgments}

This study was supported by the project of Science and Technology of Henan Province (Social Development), Research on the Integrated Development of Physical Exercise and Physical Therapy from the Perspective of Multiagent Collaboration (no. 202102310651), in 2020.

\section{References}

[1] L. Song and J. Huang, "Design of teaching quality evaluation model based on BP neural network," Electromechanical technology, vol. 19, no. 5, pp. 1-10, 2020.

[2] L. Gu, "Evaluation of multimedia teaching quality based on grey relational analysis and neural network," Modern Electronic Technology, vol. 43, no. 9, pp. 191-194, 2020.
[3] J. Zhang, "Evaluation of english teaching quality based on GA optimized RBF neural network," Computer System Application, vol. 29, no. 3, pp. 171-176, 2020.

[4] $\mathrm{Y} . \mathrm{Wu}, \mathrm{C} . \mathrm{Wu}$, and $\mathrm{Y}$. Yin, "Internet teaching quality evaluation model based on evidence theory and neural network," Modern Electronic Technology, vol. 43, no. 19, pp. 183-186, 2020.

[5] Y. Li, "Design of university teaching quality evaluation model based on data mining algorithm," Modern Electronic Technology, vol. 43, no. 17, pp. 127-130, 2020.

[6] L. Zhang, F. Zheng, L. Wang et al., "Research and application of curriculum quality evaluation system based on BP neural network," Journal of Chifeng University (Natural Science Edition), vol. 23, no. 5, pp. 13-18, 2020.

[7] Z. Zhang, Y. Lang, and Z. Xie, "Research on real time emotion recognition based on exception in classroom quality analysis," Automation Technology and Application, vol. 39, no. 6, pp. 52-57, 2020.

[8] G. Zuo, X. Su, H. Wang et al., "Research on classroom behavior detection of college students based on CNN deep learning model," Intelligent Computer and Application, vol. 10, no. 2, pp. 158-160, 2020.

[9] H. Cui, Research on the Application of K-Modes Algorithm and Neural Network in Teaching Evaluation and Learning Prediction, vol. 15, no. 2, pp. 56-63, 2020.

[10] L. Zhang, F. Zheng, L. Wang et al., "Research and application of curriculum quality evaluation system based on BP neural network," Journal of Chifeng University (Natural Science Edition), vol. 036, no. 1, pp. 102-104, 2020.

[11] Q. Wu, L. Qiang, and X. Guan, "Optical score recognition method based on multi-scale residual convolution neural network and bidirectional simple cycle unit," Progress in Laser and Optoelectronics, vol. 57, no. 8, 2020.

[12] X. Lian, J. Huang, and J. Wu, "Case study on practical teaching of neural network theory and application," Computer and Information Technology, vol. 28, no. 6, pp. 71-74, 2020.

[13] L. Shi, C. Cheng, and S. Yi, "Research on the evaluation of college students' practical teaching effect based on BP neural network," Journal of Yangzhou University (Higher Education Research Edition), vol. 24, no. 2, pp. 115-121, 2020.

[14] P. Su, "A feedback teaching mode based on machine learning," Curriculum Education Research, vol. 22, no. 14, 2020.

[15] G. Cheng, Y. Xu, and Y. Zheng, "Exploration of metalworking practice teaching reform based on machine vision to improve practice safety," Experimental Technology and Management, vol. 37, no. 5, pp. 265-269, 2020.

[16] Z. Yuanyi and L. Zheng, "Interactive text classification method of online collaborative learning based on deep neural network," Modern Distance Education Research, vol. 32, no. 3, pp. 106-114, 2020.

[17] A. Semnani, M. Ostadhassan, Y. Xu, M. Sharifi, and B. Liu, "Joint optimization of constrained well placement and control parameters using teaching-learning based optimization and an inter-distance algorithm," Journal of Petroleum Science and Engineering, vol. 203, Article ID 108652, 2021.

[18] A. Khosravi, M. Malekan, J. J. G. Pabon, X. Zhao, and M. E. H. Assad, "Design parameter modelling of solar power 
tower system using adaptive neuro-fuzzy inference system optimized with a combination of genetic algorithm and teaching learning-based optimization algorithm," Journal of Cleaner Production, vol. 244, Article ID 118904, 2020.

[19] P. Dong and A. Li, "Simulation of physical education teaching based on FPGA and wearable VR equipment," Microprocessors and Microsystems, vol. 81, Article ID 103773, 2021.

[20] N. Aelterman, M. Vansteenkiste, H. Van Keer, J. De Meyer, L. Van den Berghe, and L. Haerens, "Development and evaluation of a training on need-supportive teaching in physical education: qualitative and quantitative findings," Teaching and Teacher Education, vol. 29, pp. 64-75, 2013.

[21] J. Cheng, "Evaluation of physical education teaching based on web embedded system and virtual reality," Microprocessors and Microsystems, vol. 83, Article ID 103980, 2021.

[22] D. J. Sturm, J. Bachner, D. Renninger, S. Haug, and Y. Demetriou, "A cluster randomized trial to evaluate needsupportive teaching in physical education on physical activity of sixth-grade girls: a mixed method study," Psychology of Sport and Exercise, vol. 54, Article ID 101902, 2021. 